\title{
Beam dynamics studies for transverse electromagnetic mode type rf deflectors
}

\author{
Shahid Ahmed, ${ }^{1, *}$ Geoffrey A. Krafft, ${ }^{1,2}$ Kirsten Deitrick, ${ }^{3}$ Subashini U. De Silva, ${ }^{1,2}$ Jean R. Delayen, ${ }^{1,2}$ \\ Mike Spata, ${ }^{1}$ Michael Tiefenback, ${ }^{1}$ Alicia Hofler, ${ }^{1}$ and Kevin Beard ${ }^{4}$ \\ ${ }^{1}$ Thomas Jefferson National Accelerator Facility, Newport News, Virginia 23606, USA \\ ${ }^{2}$ Center for Accelerator Science, Old Dominion University, Norfolk, Virginia 23529, USA \\ ${ }^{3}$ Rensselaer Polytechnic Institute, Troy, New York 12180, USA \\ ${ }^{4}$ Muons, Inc., Batavia, Illinois 60510, USA \\ (Received 25 July 2011; published 14 February 2012)
}

\begin{abstract}
We have performed three-dimensional simulations of beam dynamics for transverse electromagnetic mode (TEM) type rf deflectors: normal and superconducting. The compact size of these cavities as compared to the conventional $\mathrm{TM}_{110}$ type structures is more attractive particularly at low frequency. Highly concentrated electromagnetic fields between the parallel bars provide strong electrical stability to the beam for any mechanical disturbance. An array of six 2-cell normal conducting cavities or a single cell superconducting structure is enough to produce the required vertical displacement at the target point. Both the normal and superconducting structures show very small emittance dilution due to the vertical kick of the beam.
\end{abstract}

DOI: 10.1103/PhysRevSTAB.15.022001

PACS numbers: 29.27.- a

\section{INTRODUCTION}

The Continuous Electron Beam Accelerator Facility (CEBAF) at Jefferson Lab is in the process of an energy upgrade from 6 to $12 \mathrm{GeV}$; the schematic is shown in Fig. 1. The $1497 \mathrm{MHz}$ continuous electron beam is composed of three interlaced variable intensity $499 \mathrm{MHz}$ beams that can be independently directed from any of the five distinct passes to any of the three existing experimental halls A, B, and C. Beam extraction in the existing setup is done with a system consisting of ten warm rf separator cavities; a series array of three on the 5th pass is capable of sending highest energy $(6 \mathrm{GeV})$ beams to the three experimental halls simultaneously. The undeflected beam follows a straight path to hall B and is considered reference for measuring deflection/displacement of beams traveling to halls $\mathrm{A}$ and $\mathrm{C}$. The $12 \mathrm{GeV}$ option requires ten additional cryomodules and an arc to direct the highest energy $(12 \mathrm{GeV}) 5 \frac{1}{2}$ pass beam to a newly constructed experimental hall D. The existing setup of the deflectors in the 5th pass, however, will not be adequate to extract the $11 \mathrm{GeV}$ beam in the case of the proposed $12 \mathrm{GeV}$ upgrade of the machine. To restore this capability, several options including the extension of existing CEBAF normal conducting (NC) structures or a potential $499 \mathrm{MHz}$ TEM-type superconducting (SC) design [1] are under investigation. The detailed electromagnetic characterizations of the TEM-type superconducting structure have been confirmed

\footnotetext{
*sahmed@jlab.org
}

Published by the American Physical Society under the terms of the Creative Commons Attribution 3.0 License. Further distribution of this work must maintain attribution to the author(s) and the published article's title, journal citation, and DOI. by the three-dimensional simulations $[1,2]$. Also, the rigorous analysis of the existing CEBAF normal conducting cavity from the rf design viewpoint has been reported in $[3,4]$ and, more importantly, the device is presently operating. These rf separator cavities (SC/NC) are supposed to provide deflections of $\sim \pm 400 \mu \mathrm{rad}$ for $11 \mathrm{GeV}$ beams traveling to halls $\mathrm{A}$ and $\mathrm{C}$ in the $12 \mathrm{GeV}$ machine. The rf kick is determined by the requirement to have the beams to halls $\mathrm{A}$ and $\mathrm{C}$ vertically separated by $\pm 17 \mathrm{~mm}$ relative to hall $\mathrm{B}$ at the entrance of the existing extraction magnet (Lambertson style) in the beam switch-yard located $43 \mathrm{~m}$ downstream from the start of the separator cavity. This work is the continuation of the first study reported in [5]. In this paper, we report the beam dynamics studies for $499 \mathrm{MHz}$ TEM-type rf deflecting cavities (see Figs. 2 and 4) for the 5th pass $(11 \mathrm{GeV})$ beam in the case of $12 \mathrm{GeV}$ upgrade of CEBAF at JLab.

The following are the important issues that need to be addressed in order to conclude that the cavities are suitable.

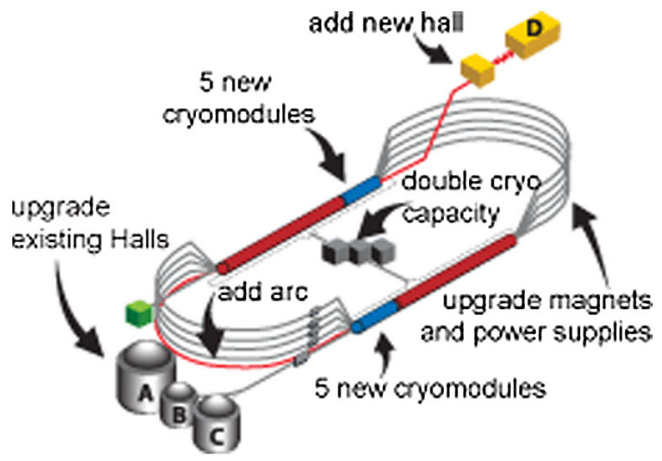

FIG. 1. Continuous Electron Beam Accelerator Facility (CEBAF) $12 \mathrm{GeV}$ upgrade schematic. 


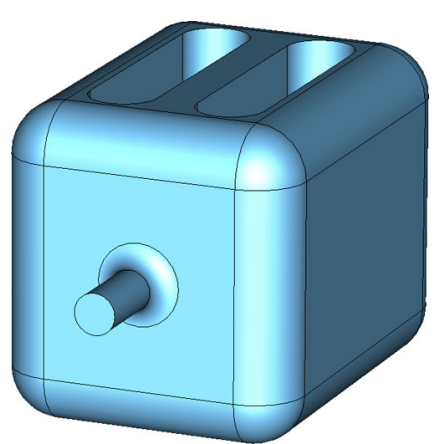

FIG. 2. Parallel-bar superconducting rf cavity geometry as illustrated in [2].

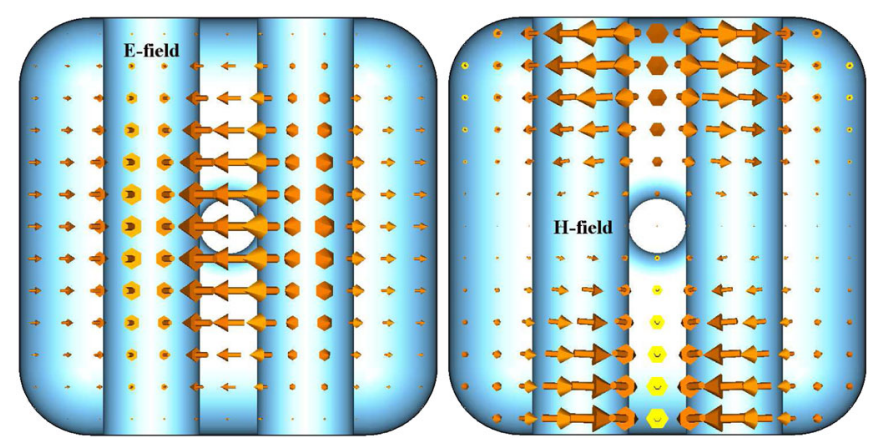

FIG. 3. Electromagnetic field $(\vec{E}, \vec{H})$ distribution of the superconducting cavity (see Fig. 2) in the $x y$ plane. The electric field is strongly concentrated over the central region due to the dominant capacitive effect; however, the magnetic field becomes predominant near the top and bottom where the electrodes are electrically shorted with the corresponding perfectly conducting plates.

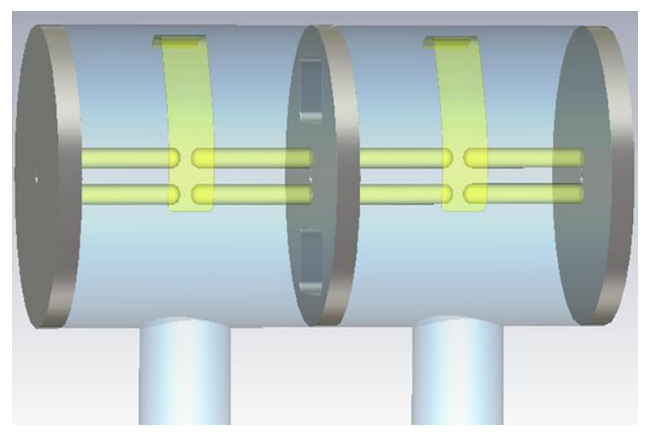

FIG. 4. Schematic of 2-cell normal conducting cavity as discussed in [4].

First, ensure that there is no strong nonlinear effect of electromagnetic (EM) fields inside the new superconducting structure disturbing the particle trajectories to the experimental stations. Second, investigate any nonzero residual orbit offset for the reference beam directed to hall $\mathrm{B}$ at the final exit of the cavity and explore its consequences. Because all the measurements are relative to hall B beam, any offset in its position will affect the required vertical displacements to the beams directed to halls $\mathrm{A}$ and $\mathrm{C}$. Third, the beam stay clear condition imposes a constraint of a $6.5 \mathrm{~mm}$ gap from either end of the pipe wall to avoid beam loss on the beam pipe aperture. Fourth, the horizontal and transverse misalignments for an array of normal conducting cavities should not exceed $1 \mathrm{~mm}$. The electromagnetic fields are concentrated within the narrow aperture of the normal conducting cavity. Therefore, a significant misalignment of cavities may lead to insufficient kick, which, in turn, may cause beam loss. Fifth, the projected normalized rms emittance dilution should be less than $1 \mathrm{~mm}$ mrad. This limits the beam size under the experimental requirement and avoids unwanted effects. The details of these studies are discussed in the subsequent sections and the organization of the paper is illustrated below.

Section II gives the details of the model with the description of numerical methods used in this study. Section III summarizes the benchmark of the computer programs. In Sec. IV, the physics of nonzero residual orbit offset corresponding to the reference rf phase is discussed. Section V describes the details of superconducting structure, and comprehensive studies of the warm cavity option are explored in Sec. VI. Finally, the concluding remarks of this study are summarized in Sec. VII.

\section{MODEL DESCRIPTION}

In this section, configurations of the structures and their electromagnetic properties are discussed briefly, followed by the discussions on the particle tracking methods and the computer programs used.

\section{A. Radio-frequency structures}

The configurations of the cavities considered in this study are shown in Figs. 2 and 4. The details of these cavities from the electromagnetic design viewpoints are well documented in [2-4], however, we will discuss briefly for the sake of completeness. The superconducting structure consists of a beam pipe, passing between the two perpendicular $\lambda / 2$ parallel rods as shown in Fig. 2. The rods are oriented along the $y$ direction (shown vertical) and separated along the $x$ direction with a beam pipe extended along the $z$ direction. In the deflecting mode of operation, the two rods resonate in opposite phase ( $\pi$ mode). This sets up a strong transverse electromagnetic field (TEM) field between the rods-see Fig. 3.

There are eight rods (each $\lambda / 4$ long) in a single unit (2-cell) of normal conducting structure, in which a pair of rods is separated vertically along the $x$ direction and placed longitudinally along the $z$ direction (i.e. the beam line)see Fig. 4. The parallel rods effectively reduce the transverse dimension and confine the EM fields into the central region (see Fig. 5) of the cavity [3]. Two rectangular openings at the midplate provide EM coupling between the two halves. This full-wave structure reduces the power 


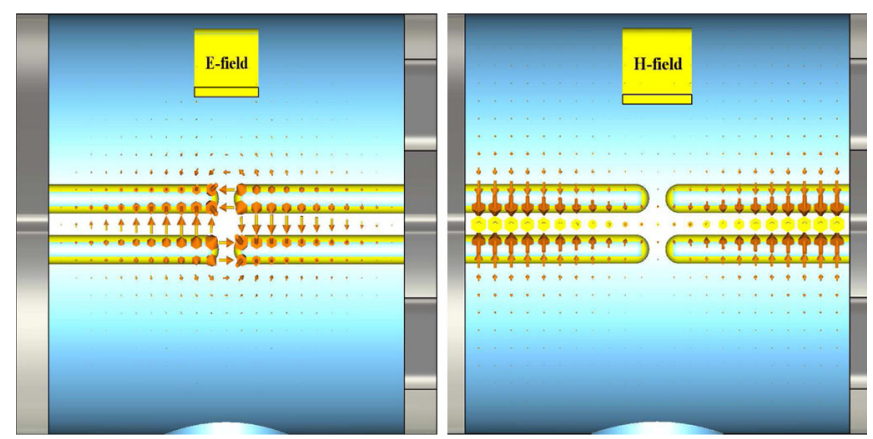

FIG. 5. Electromagnetic field $(\vec{E}, \vec{H})$ distribution in the $x z$ plane of the 1-cell of cavity shown in Fig. 4.

requirement by $50 \%$ as compared to a half-wave structure for the same deflection [6]. A capacitive tuner has been mounted in order to tune the cavity at the correct frequency, which is shown by yellow flat strip in Fig. 4. This consists of a copper flap, grounded to the wall of the cavity on one end and bent to the close proximity of the rods. A comparison of the basic properties of superconducting (see Fig. 2) and normal conducting (see Fig. 4) cavities are summarized in Table I.

\section{B. Numerical scheme for particle tracking}

To assure the correctness of the simulations, the beam dynamics studies have been carried out numerically by three different packages-general particle tracer (GPT) [7], G4BEAMLINE [8], and CST PARTICLE TRACKING SIMULATOR [9]. The GPT and G4BEAMLINE require field maps for tracking particles, which is obtained from the eigenmode solver of CST MICROWAVE STUDIO. GPT is a well established three-dimensional time domain computer

TABLE I. Properties of structures as shown in Figs. 2 and 4.

\begin{tabular}{lcc}
\hline \hline Parameters & SC (Fig. 2) & NC (Fig. 4) \\
\hline Frequency of $\pi$ mode $(\mathrm{MHz})$ & 499.2 & 499 \\
$\lambda / 2$ of $\pi$ mode $(\mathrm{mm})$ & 300.4 & 300.4 \\
Frequency of 0 mode $(\mathrm{MHz})$ & 517.8 & 537 \\
Cavity length $(\mathrm{mm})$ & 394.4 & 300 \\
Cavity width $(\mathrm{mm})$ & 290 & 292 \\
Bars height $(\mathrm{mm})$ & 304.8 & 20 \\
Bars width $(\mathrm{mm})$ & 67 & 20 \\
Bars length $(\mathrm{mm})$ & 284 & 135 \\
Aperture diameter $(\mathrm{mm})$ & 40 & 15 \\
Deflecting voltage $\left(V_{T}^{*}\right)(\mathrm{MV})$ & 0.3 & 0.3 \\
Peak $E$ field $\left(E_{P}^{*}\right)(\mathrm{MV} / \mathrm{m})$ & 1.85 & 3.39 \\
Peak $B$ field $\left(B_{P}^{*}\right)(\mathrm{mT})$ & 6.69 & 8.87 \\
Energy content $\left(U^{*}\right)(\mathrm{mJ})$ & 31 & 1.2 \\
Geometric factor $(\Omega)$ & 67.96 & 34.9 \\
$(R / Q)_{T}(\Omega)$ & 933.98 & 24921 \\
rf field amplitude tolerance $\delta A / A$ & \multicolumn{2}{c}{$0.1 \%$} \\
rf phase tolerance $\delta \phi$ & $<1^{\circ}$ \\
at $E_{T}^{*}=1 \mathrm{MV} / \mathrm{m}$ & \multicolumn{2}{|}{} \\
\hline \hline
\end{tabular}

program for studying particle dynamics in EM fields. The tracking algorithm is based on the fifth order Runge-Kutta method with adaptive step size and takes into account the space charge physics and other nonlinearities. G4BEAMLINE is a reliable and robust computer program based on GEANT4 [10]. GEANT4 tracks a particle by integrating the equations of motion in an electromagnetic field using 4th-order Runge-Kutta integrator with variable step size; multiple scattering and ionization energy loss are applied to each step. The physics step is limited by the shortest interaction length of all physics processes configured for the particle being tracked; whichever process limited the step is then invoked at the end of step. There are multiple integration steps within a physics step based on the curvature of the track. In the latest development, G4BEAMLINE includes multiple particle tracking in time and space charge physics. Moreover, it provides visualization of the simulated objects and tool for graphics. On the other hand, the particle tracking simulator of CST MICROWAVE STUDIO is a new development, however, provides much flexibility for studying beam dynamics of a complex object. The tracking algorithm is based on the integration of the equations of motion in an electromagnetic field using a leapfrog method (centered difference) with dynamic time-step size of integration.

\section{Determination of operating rf phase}

To determine the operating phase numerically, we compute the deflection at the exit of the cavity for one complete rf cycle $\phi_{\mathrm{rf}}=0^{\circ}$ to $360^{\circ}$ as shown in Fig. 6 . As the cavity is half-wave long, we expect the deflection following cosine function. The first zero crossing at $\phi_{\mathrm{rf}}=90^{\circ}$ corresponds to the zero deflection and is defined as the reference phase $\phi_{\text {ref }}$. As the hall B beam is undeflected (directed straight), this reference phase is the representative of the beam going to hall B, i.e. $\phi_{B}=\phi_{\text {ref }}$. Therefore,

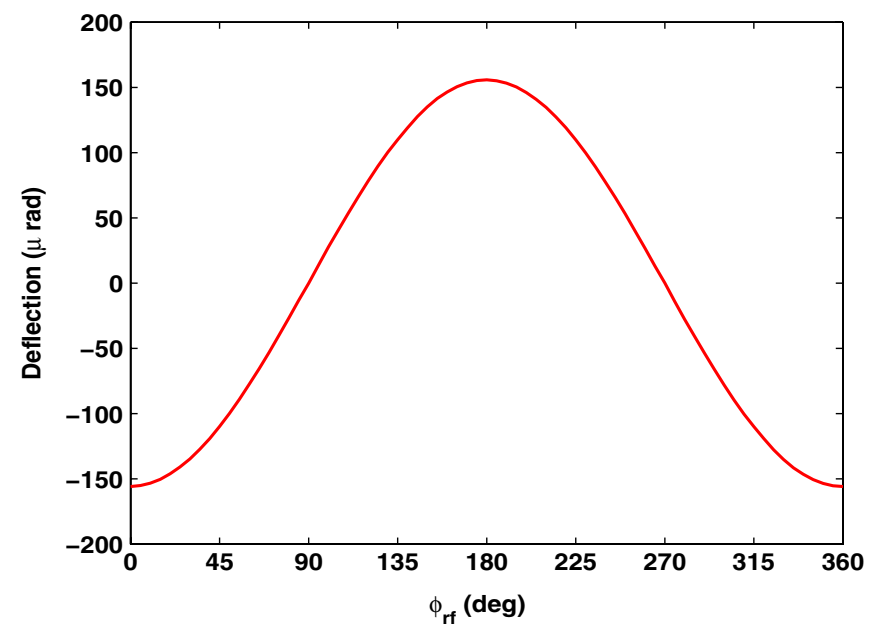

FIG. 6. Vertical deflection versus rf phase at the exit of the cavity shown in Fig. 2. 
the corresponding phases of the beam directed to halls A and C (see Fig. 1) are given by $\phi_{A, C}=\phi_{B} \pm 120^{\circ}$. This phase convention will be used throughout this study unless stated explicitly.

\section{BENCHMARK OF SIMULATION}

Before starting the detailed simulations of the actual problem, it is always advisable to verify the correctness of the computer programs used. For this purpose, we track an $11 \mathrm{GeV}$ electron on axis of the superconducting structure as shown in Fig. 2 and compare the results obtained from CST PARTICLE TRACKER, GPT, and G4BEAMLINE computer programs. Figure 7 shows vertical deflection $\left(p_{x} / p_{z}\right)$

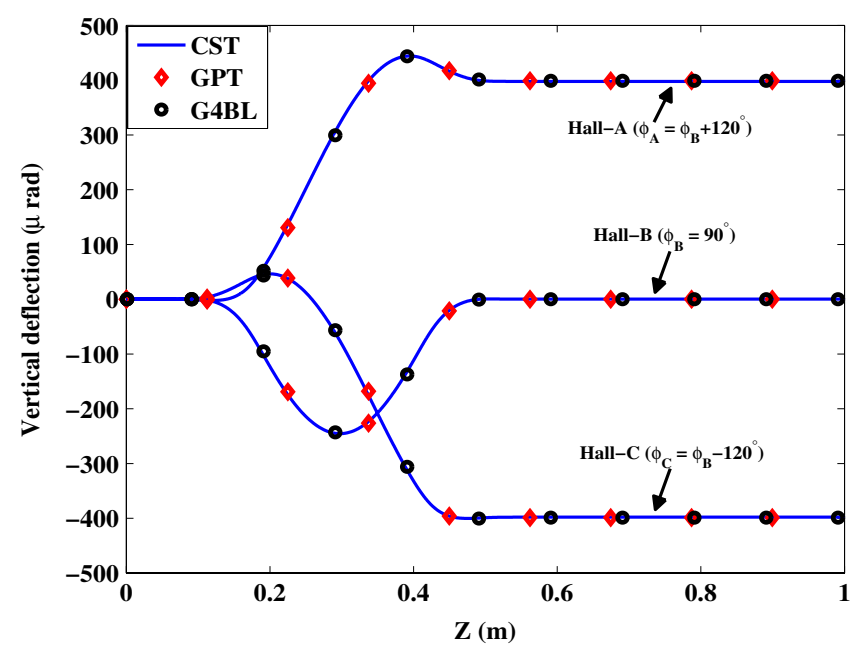

FIG. 7. Vertical deflection of an $11 \mathrm{GeV}$ electron for rf phases $\phi_{A}, \phi_{B}$, and $\phi_{C}$ of the superconducting cavity (see Fig. 2). Simulation results are compared using CST PARTICLE TRACKER, GPT, and G4BEAMLINE computer codes.

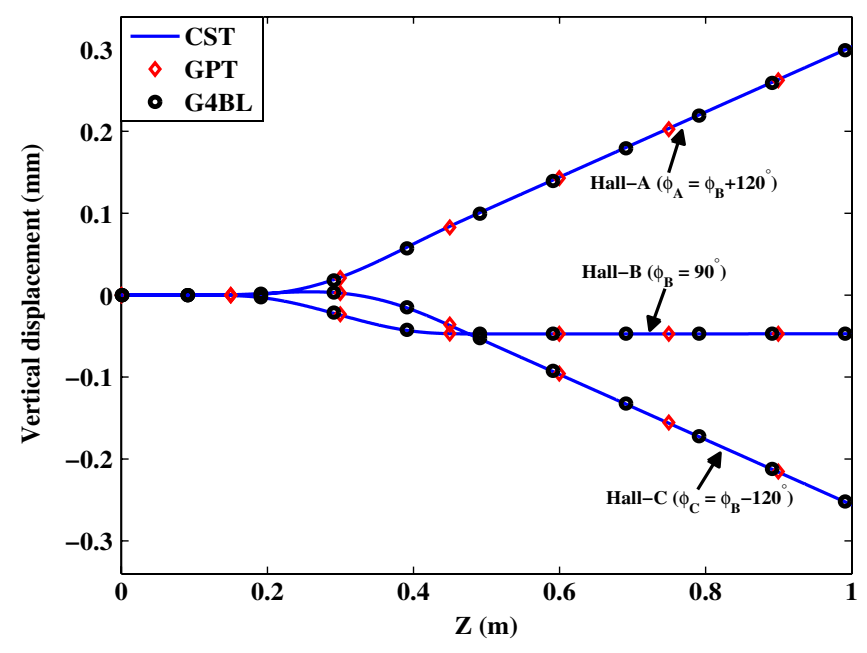

FIG. 8. Comparison of vertical displacement results using CST PARTICLE TRACKER, GPT, and G4BEAMLINE computer codes. Parameters chosen are the same as in Fig. 7. of the particle corresponding to the three different phases $\phi_{A}, \phi_{B}$, and $\phi_{C}$ as discussed earlier. The corresponding displacements of the particle are shown in Fig. 8. The following points are important to note. First, the results obtained from the CST PARTICLE TRACKER, GPT, and G4BEAMLINE simulation programs show excellent agreement. Second, the deflection of the beam directed to hall B is zero and constant to halls $\mathrm{A}$ and $\mathrm{C}$ after leaving the cavity $(z=60 \mathrm{~cm})$. Third, simulations show nonzero residual orbit offset $(\sim 47 \mu \mathrm{m})$ corresponding to the undeflected beam ( $\phi=\phi_{B}$, see Fig. 8).

\section{NONZERO RESIDUAL ORBIT OFFSET FOR REFERENCE RF PHASE}

In the previous section, we have seen a nonzero residual orbit offset corresponding to the reference rf phase $\phi_{B}$ (see Fig. 8). This effect is physical and arises due to the finite transit time of beam through a thick rf deflector. It is therefore important to understand the mechanism so that the effect could be manipulated if required. Let us consider a simple case of an ideal cylindrically symmetric pillbox cavity without beam pipe which can be solved analytically. We consider the particle dynamics corresponding to the reference $\mathrm{rf}$ phase. The deflecting angle and the vertical displacement at the exit of a thick $(L)$ cavity can be expressed as [11]

$$
\begin{gathered}
x_{f}^{\prime}=x_{0}^{\prime}+\frac{q_{e} V_{\mathrm{def}}}{E_{b}} \sin \phi \\
x_{f}=x_{0}+\frac{L}{2}\left(x_{0}^{\prime}+x_{f}^{\prime}\right)+\frac{q_{e} V_{\mathrm{def}}}{\kappa E_{b}} \cos \phi,
\end{gathered}
$$

where $q_{e}\left(=1.6 \times 10^{-19} \mathrm{C}\right), V_{\text {def }}(\sim 5.06 \mathrm{MV}), E_{b}(=11 \mathrm{GeV})$, $\kappa\left(=2 \pi / \lambda \sim 10.45 \mathrm{~m}^{-1}\right), x_{0}(=0), x_{0}^{\prime}(=0), x_{f}$, and $x_{f}^{\prime}$ are the electronic charge, rf deflecting voltage, beam energy, wave number, initial position, initial angle, final position, and final angle, respectively. Note that the reference phase in the above equations (1) and (2) is $\phi_{\text {ref }}=0^{\circ}$ and other $\mathrm{rf}$ phases are measured relative to the reference.

For quantitative understanding, we have compared the superconducting structure (see Fig. 2) with the full-wave 3D analysis of a pillbox with beam pipe and the idealized pillbox cavity without beam pipe operating at $499 \mathrm{MHz}$. The major difference between the pillbox cavity and the superconducting structure are the EM modes of operation, which are the $\mathrm{TM}_{110}$ and the TEM, respectively. In other words, the deflection in the case of the pillbox cavity is mainly contributed by the magnetic field, however, the electric field plays an important role in the case of the superconducting structure. This exercise is aimed to quantify the difference between the realistic and the idealized structures operating in different EM modes. This study can be significantly important in precision measurements of the order of few 100 microns. The tracking studies of an $11 \mathrm{GeV}$ electron near to the axis of the cavities at $\phi=0$ 

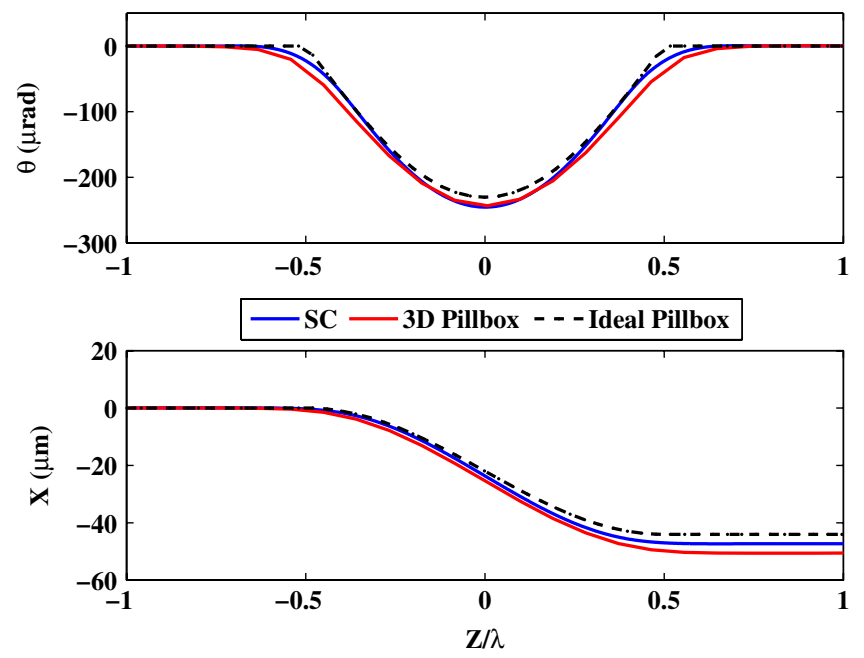

FIG. 9. Comparison of deflecting angle $(\theta)$ and vertical displacement $(X)$ experienced by an $11 \mathrm{GeV}$ electron propagating near the axis of the cavities for the reference rf phase $\phi=0^{\circ}$. The acronyms "SC", "3D Pillbox", and "Ideal Pillbox" correspond to the superconducting structure, full-wave 3D EM model of pillbox with beam pipe, and an idealized pillbox cavity without beam pipe, respectively.

have been performed. The deflection and the displacement observed while propagating inside the cavities are compared and illustrated in Fig. 9. The legends showing "SC," "3D Pillbox," and "Ideal Pillbox" correspond to the superconducting structure, full-wave 3D analysis of pillbox with beam pipe, and an ideal pillbox cavity without beam pipe, respectively. The deflection at the exit of the cavity is perfectly zero for all three cases, which is consistent with the derivation shown in (1). The vertical displacements $\sim 47.3 \mu \mathrm{m}$ for (SC), $50 \mu \mathrm{m}$ (3D pillbox), and $44 \mu \mathrm{m}$ (ideal pillbox) showing nonzero residual orbit offset. For an ideal pillbox, the numerical value of $44 \mu \mathrm{m}$ agrees very well with the expected offset of $43.97 \mu \mathrm{m}$, however, the full-wave simulations are within (7-13)\%. The higher values in the case of full-wave threedimensional simulations are due to the real field distributions including edge effects.

\section{BEAM STUDIES FOR SUPERCONDUCTING STRUCTURE}

In this section, we discuss the beam dynamics of the superconducting structure.

\section{A. Displacement and deflection at the Lambertson magnet}

The requirement is to produce a vertical displacement of $17 \mathrm{~mm}$ for beams to halls $\mathrm{A}$ and $\mathrm{C}$ at a distance of $43 \mathrm{~m}$ where the Lambertson magnet is located. This criterion has been set by the location of the aperture of the Lambertson magnet.

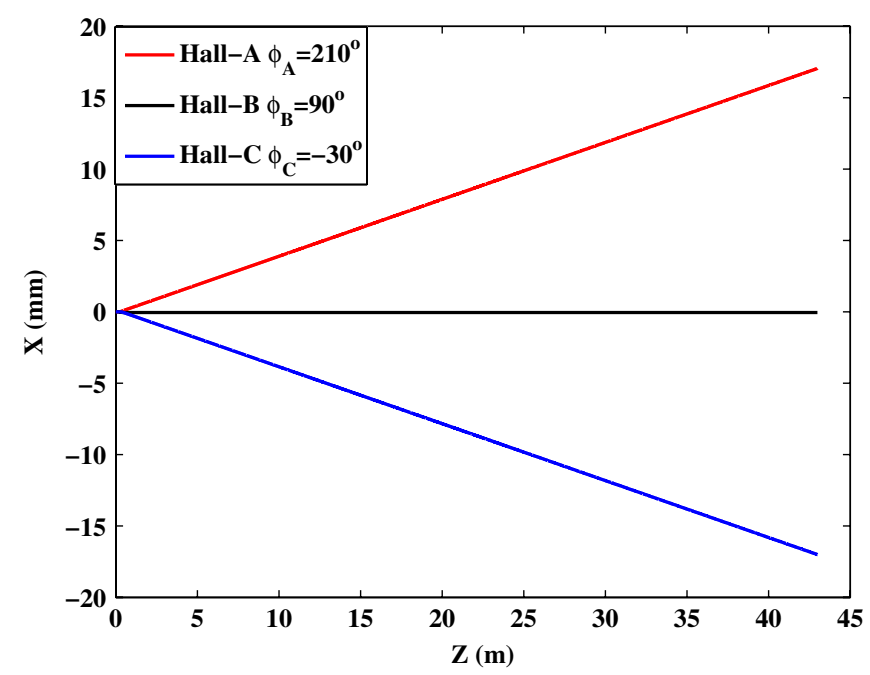

FIG. 10. Evolution of vertical displacement corresponding to the rf phases $\phi_{A, B, C}$ and deflecting voltage $V_{\mathrm{def}}=5.05 \mathrm{MV}$.

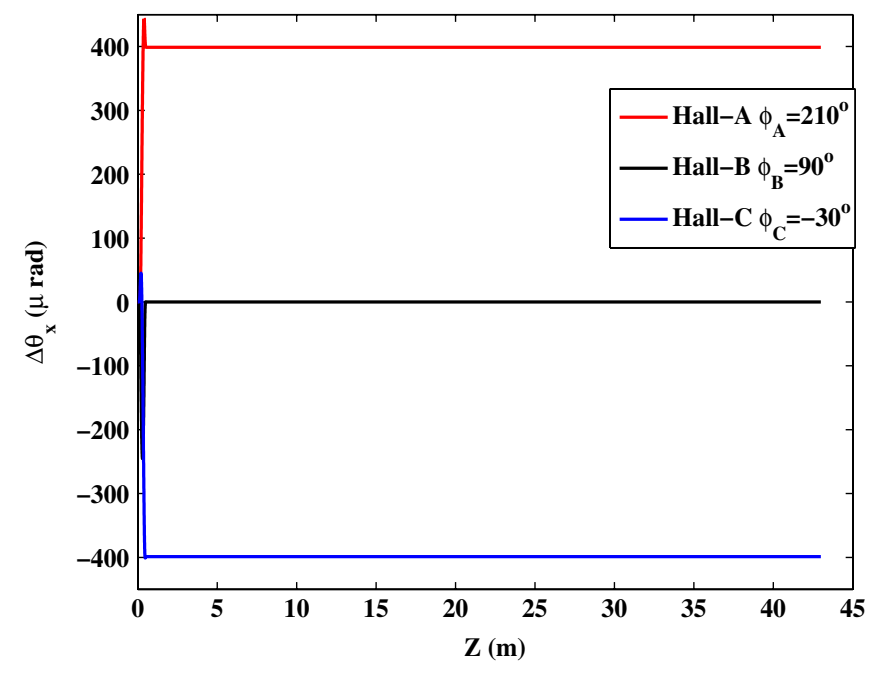

FIG. 11. Evolution of vertical deflection corresponding to $\mathrm{rf}$ phases $\phi_{A, B, C}$ and deflecting voltage $V_{\text {def }}=5.06 \mathrm{MV}$.

Figures 10 and 11 show the evolution of vertical displacement and deflection $43 \mathrm{~m}$ downstream of the cavity where the extraction magnet is located. The nature of deflecting angle (see Fig. 11) at the exit of the cavity is consistent with the earlier observation in Fig. 7. However, the residual nonzero orbit offset and other nonlinearities (asymmetries in tracks) observed in close proximity to the cavity become relatively unimportant in the far field ( $\sim 43 \mathrm{~m}$ downstream). This is because these effects are significantly small as compared to the vertical displacement of $17 \mathrm{~mm}$ observed at the entrance of the extraction magnet. So, the nonzero residual orbit offset is not an issue in this type of cavity in this application. We see three distinct orbits corresponding to the three different rf phases $\phi_{A, B, C}$ for beams directed to the experimental stations $A, B$, and $C$, respectively. This confirms that the nonlinear effects 
due to the 3D EM fields inside the cavity are too weak to affect the beam dynamics.

\section{B. Beam emittance}

In previous sections, we have performed the single particle tracking to establish the understanding of deflection and displacement due to a deflecting cavity. In this section we will study the effect resulting in the propagation of a bunch of particles. We start our simulation by considering the uniform distribution of electrons in a bunch with total number of particles $N_{p}=1000$, total charge $Q_{\text {tot }}=$ $1 \mathrm{pC}$, beam energy $E_{b}=11 \mathrm{GeV}$, bunch radius $r_{b}=$ $100 \mu \mathrm{m}$, and bunch length $l_{b}=100 \mu \mathrm{m}$.

Figure 12 shows the evolution of normalized projected emittance along the $z$ direction of beam propagation. We observe the projected emittance growth primarily along the $x$ direction which is due to the dominant deflecting force, however, the other components are significantly small. Also, we observe that the projected emittance of the undeflected beam is twice as much as the deflected one. This is because the slope of the deflecting voltage at $\phi_{B}$ is double to that of $\phi_{A, C}$. The following analytical derivation has been made for the consistency of the numerical result.

The transverse force along the $x$ direction on a relativistic particle moving along the $z$ direction in an electromagnetic field is defined as

$$
\frac{d p_{x}}{d z}=\frac{q_{e}}{c}\left(E_{x}+c B_{y}\right) \Rightarrow p_{x}=\frac{q_{e}}{c} V_{\mathrm{def}},
$$

where $V_{\text {def }}$ is the net deflecting voltage. We can rewrite the above equation by considering the rf phase as follows:

$$
p_{x}=\frac{q_{e}}{c} V_{\text {def }} \sin (\kappa z),
$$

where the wave number $\kappa=\frac{2 \pi}{\lambda}$. For bunch length smaller than the rf wavelength, Eq. (4) reduces to
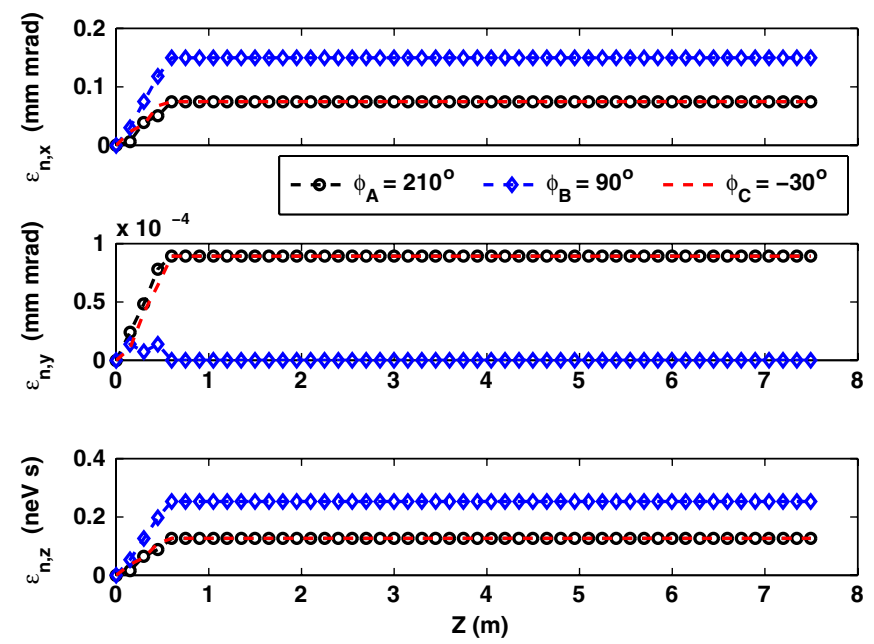

FIG. 12. Evolution of normalized projected rms emittance $\left[\epsilon_{n,(x, y, z)}(z)\right]$ corresponding to the rf phases $\phi_{A, B, C}$ along the direction of beam propagation.

$$
p_{x}=\frac{q_{e}}{c} V_{\mathrm{def}}(\kappa z)
$$

Let us consider a uniform distribution of particles inside a bunch of cylindrical shape having radius $r_{b}$ and length $l_{b}$. The distribution function is represented by

$f(x, y)=\frac{1}{\pi a^{2}} f\left(r^{2}-r_{b}^{2}\right) ; \quad f\left(r^{2}-r_{b}^{2}\right)= \begin{cases}1 & \text { if } r \leq r_{b} \\ 0 & \text { if } r>r_{b},\end{cases}$

where $r=\sqrt{\left(x^{2}+y^{2}\right)}$ and in polar coordinate $x=r \cos \theta$, $y=r \sin \theta$. Now $\langle x\rangle$ and $\left\langle x^{2}\right\rangle$ are computed as

$$
\begin{gathered}
\langle x\rangle=\int_{0}^{\infty} \int_{0}^{\infty} x f(x, y) d x d y=\int_{0}^{2 \pi} \int_{0}^{r_{b}} \frac{r^{2}}{\pi a^{2}} d r \cos \theta d \theta=0 \\
\left\langle x^{2}\right\rangle=\int_{0}^{\infty} \int_{0}^{\infty} x^{2} f(x, y) d x d y \\
=\int_{0}^{2 \pi} \int_{0}^{r_{b}} \frac{r^{3}}{2 \pi a^{2}}(1+\cos 2 \theta) d r d \theta=\frac{r_{b}^{2}}{4} \\
\left\langle p_{x}^{2}\right\rangle=\frac{q_{e}^{2}}{c^{2}} V_{\mathrm{def}}^{2} \kappa^{2} z^{2}=\frac{q_{e}^{2}}{12 c^{2}} V_{\mathrm{def}}^{2} \kappa^{2} l_{b}^{2}
\end{gathered}
$$

For a bunch uniform in the longitudinal direction with bunch length $l_{b}$, the normalized projected rms emittance is to be defined as [12]

$$
\epsilon_{n, \mathrm{rms}}^{2}=\frac{\left\langle x^{2}\right\rangle\left\langle p_{x}^{2}\right\rangle-\left\langle x p_{x}\right\rangle^{2}}{m^{2} c^{2}} .
$$

Since $\left\langle x p_{x}\right\rangle=0$, the above equation reduces to

$$
\epsilon_{n, \mathrm{rms}}^{2}=\frac{\left\langle x^{2}\right\rangle\left\langle p_{x}^{2}\right\rangle}{m^{2} c^{2}} .
$$

By substituting the values of $\langle x\rangle$ and $\left\langle p_{x}^{2}\right\rangle$, we obtain

$$
\epsilon_{n, \mathrm{rms}}=\frac{q_{e} V_{\mathrm{def}}}{4 \sqrt{3} m c^{2}} \kappa r_{b} l_{b} .
$$

The above equation (12) establishes the relationship of the normalized projected emittance increase with the beam parameters, rf frequency, and the deflecting voltage. Substituting the values of the bunch parameters and deflecting voltage $V_{\text {def }}=5.06 \mathrm{MV}$, we get the normalized projected $x$ emittance corresponding to the undeflected beam $\left(\phi=\phi_{B}\right), \epsilon_{x, n \text { rms }}=0.15 \mathrm{~mm} \mathrm{mrad}$ which is exactly the simulated value of $0.15 \mathrm{~mm}$ mrad. However, the normalized projected emittance of the deflected beams $\left(\phi=\phi_{A, C}\right)$ is half to that of the undeflected one due to half in the value of the slope of deflecting voltage.

\section{Effect of bunch length and radius}

From Eq. (12), we expect a linear dependence of projected emittance increase on the bunch length and radius. The effects are indeed confirmed by the simulationsshown in Figs. 13 and 14. 


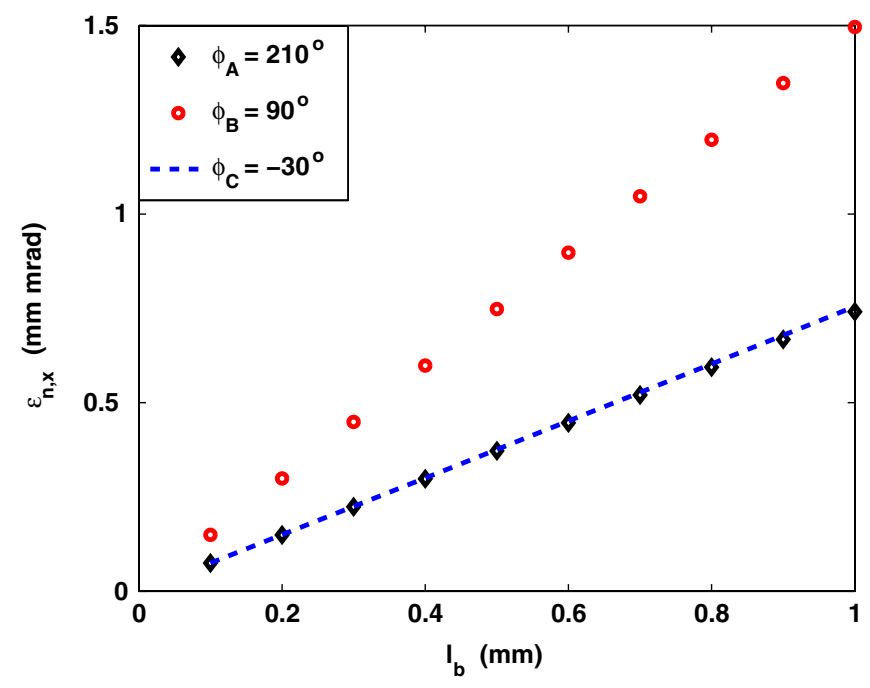

FIG. 13. Normalized rms projected emittance $\left(\epsilon_{n, x}\right)$ for different bunch length $\left(l_{b}\right)$ corresponding to rf phases $\phi_{A, B, C}$.

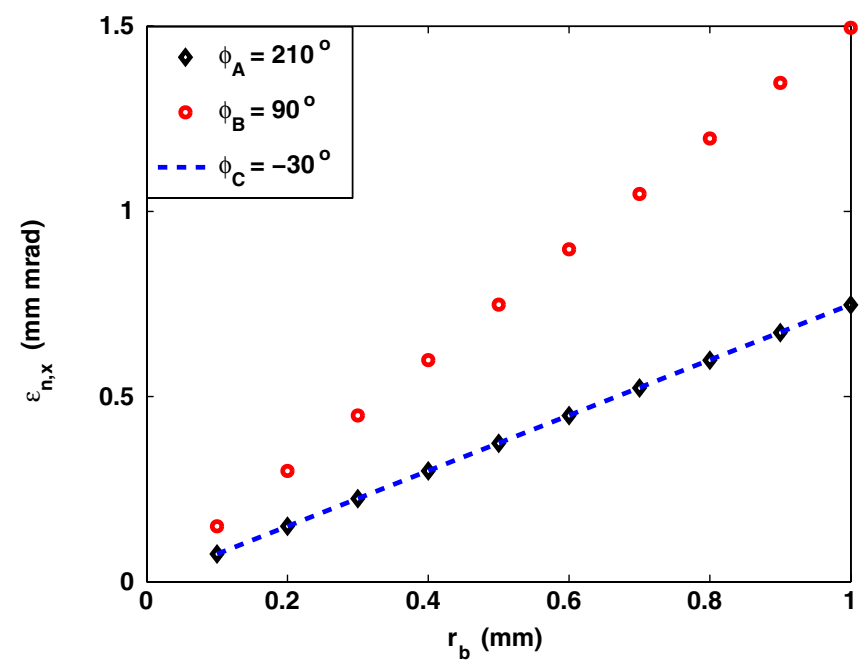

FIG. 14. Normalized rms projected emittance $\left(\epsilon_{n, x}\right)$ for different bunch radius $\left(r_{b}\right)$ corresponding to $\mathrm{rf}$ phases $\phi_{A, B, C}$.

\section{NORMAL CONDUCTING CAVITY}

In earlier sections, we have studied the beam dynamics of the superconducting structure. The superconducting cavity has advantages because of higher gradient in a compact size system, however, the cryogenics and the power consumption issues cannot be ignored. The
CEBAF normal conducting deflecting cavities in its current setup are performing well. It is therefore important to understand the physical properties of the normal conducting structure from the beam dynamics viewpoint. Because the normal conducting cavities are operated at lower field gradient, we need more than one cavity to achieve the required displacement $(17 \mathrm{~mm})$ at the Lambertson magnet.

Figure 15 shows the series arrangement of eight 2-cell cavities; each unit (2-cell) is a full wavelength $(\lambda)$ long and the drift space between the two consecutive units is gap $=$ $130.06 \mathrm{~mm}$. The center-to-center distance of $731.26 \mathrm{~mm}$ between two unit cells results in the time delay of $\Delta t=2.44 \mathrm{~ns}$, which corresponds to the phase delay of $\omega \Delta t=437.88^{\circ}$. The total distance of eight unit cells is $5.72 \mathrm{~m}$. It is important to take the proper phase delay into account to enhance the kick from each unit cell.

The tracking of an $11 \mathrm{GeV}$ electron through the cavities is shown in Figs. 16 and 17. Figure 16 shows the vertical deflection experienced by the electron. As expected the deflection from each cavity (53 $\mu \mathrm{rad})$ gets added up resulting in the final deflection of $424 \mu \mathrm{rad}$ at the exit of the final cavity. The corresponding deflecting voltage used is $673.2 \mathrm{kV}$. The vertical displacement is illustrated in Fig. 17, which is about $1.2 \mathrm{~mm}$ on one side of the pipe wall at the final exit of the cavity. This is $200 \mu \mathrm{m}$ higher than the tolerance. However, an array of six 2-cell cavities provide a vertical displacement of $800 \mu \mathrm{m}$ on one side of the pipe wall, which very well satisfies the beam clear requirement. This is very important from the beam dynamics viewpoint and provides an alternative option of the superconducting structure.

\section{A. Cavity misalignment}

The normal conducting cavity in its current design has a beam pipe diameter of $15 \mathrm{~mm}$. It is therefore possible that the misalignment of the cavities due to mechanical vibration may result in the disruption of beam. In order to understand the effect, we have performed beam dynamics studies for different random displacements of the cavities transversely. This means that the cavities are completely misaligned. In this simulation, we have studied the worst case by considering the misalignment far off the requirement. This misalignment is good enough to take into account the angular tilt of the cavities as well. Figure 18 shows the vertical displacement of an $11 \mathrm{GeV}$ electron for the transverse shift of cavities by 3 and $5 \mathrm{~mm}$ and its

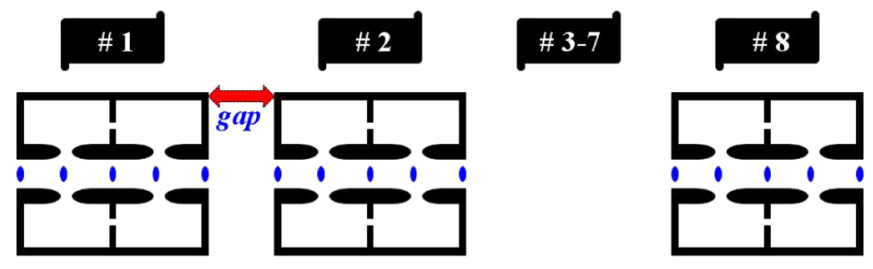

FIG. 15. Schematic of normal conducting cavity arrangement. 


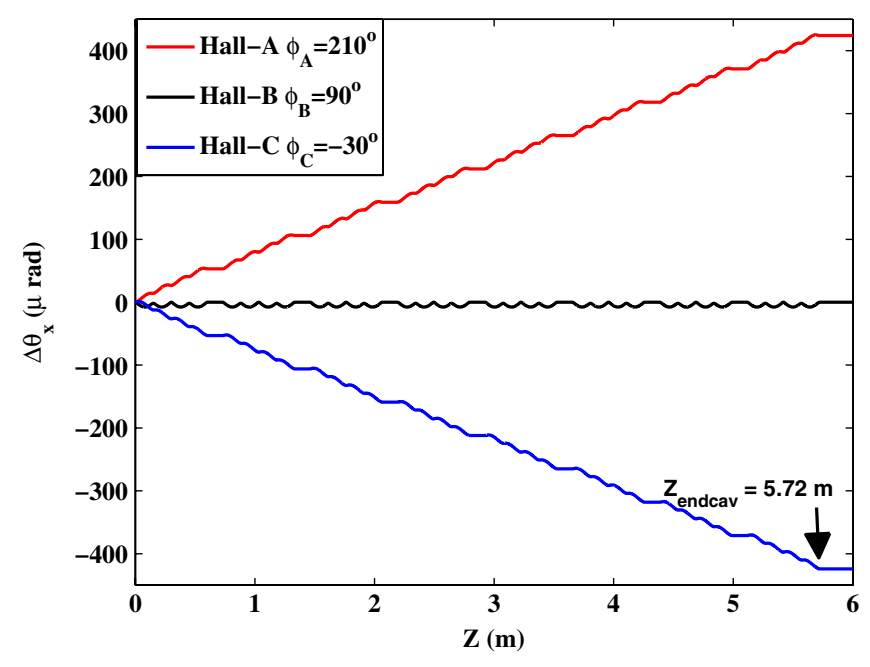

FIG. 16. Vertical deflection of an $11 \mathrm{GeV}$ electron due to eight 2-cell cavities for three different $\mathrm{rf}$ phases $\phi_{A, B, C}$ and $V_{\text {def }}=$ $673.2 \mathrm{kV}$.

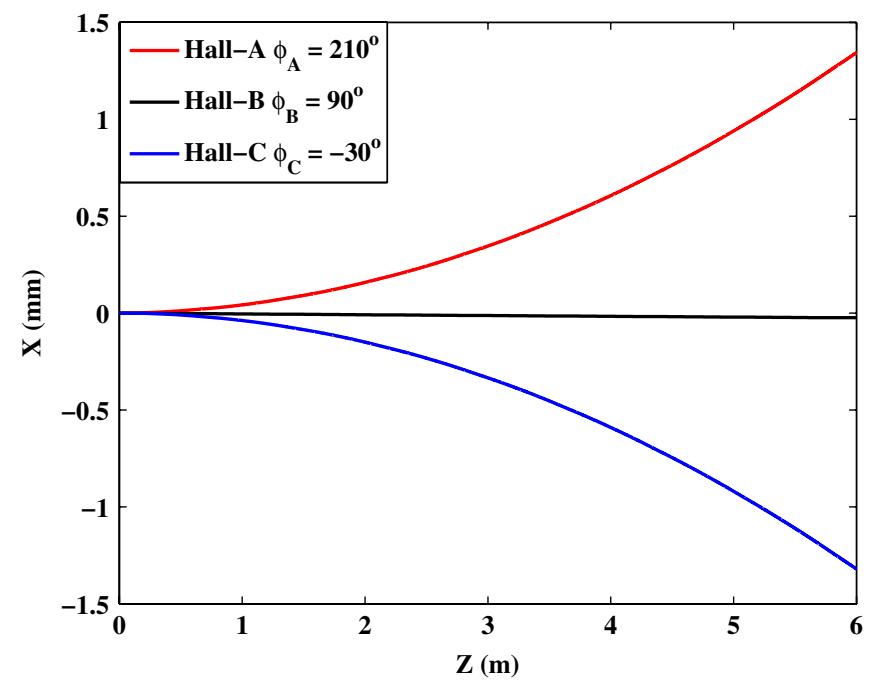

FIG. 17. Vertical displacement of an $11 \mathrm{GeV}$ electron due to eight 2-cell cavities for three different rf phases $\phi_{A, B, C}$ and $V_{\text {def }}=673.2 \mathrm{kV}$.

comparison with perfectly aligned cavities shown by the legend " $0 \mathrm{~mm}$ ". We see that the tracks for " $3 \mathrm{~mm}$ " and " $5 \mathrm{~mm}$ " misalignments are exactly the same as the perfect alignment. These simulations suggest that the particles experience the same vertical kick in the misaligned and the perfectly aligned conditions. This is because the strength of EM fields distributed over the cross section occupying between the rods is fairly constant. In brief, we conclude that the cavities are insensitive to the transverse misalignments within the aperture of the beam pipe. We have also studied the horizontal tolerance by displacing the cavities randomly along the beam line by 1 to $3 \mathrm{~mm}$. It is noticed that the vertical displacement at the exit of the final cavity is almost constant confirming the tolerance

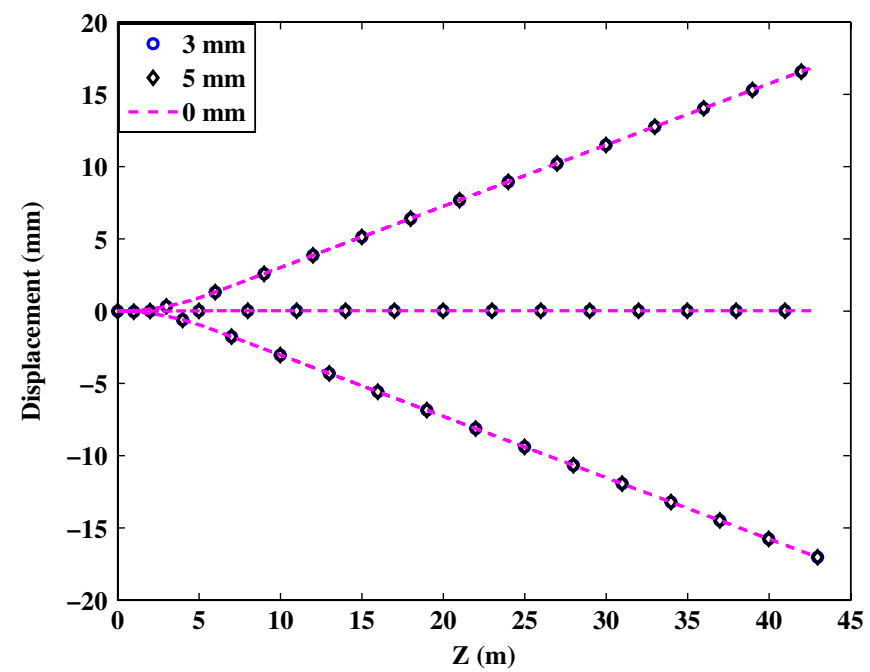

FIG. 18. Vertical displacement for an $11 \mathrm{GeV}$ electron corresponding to the transverse displacements of 3 and $5 \mathrm{~mm}$ for $\mathrm{rf}$ phases $\phi_{A, B, C}$. The legend corresponding to " $0 \mathrm{~mm}$ " refers to perfect alignment.

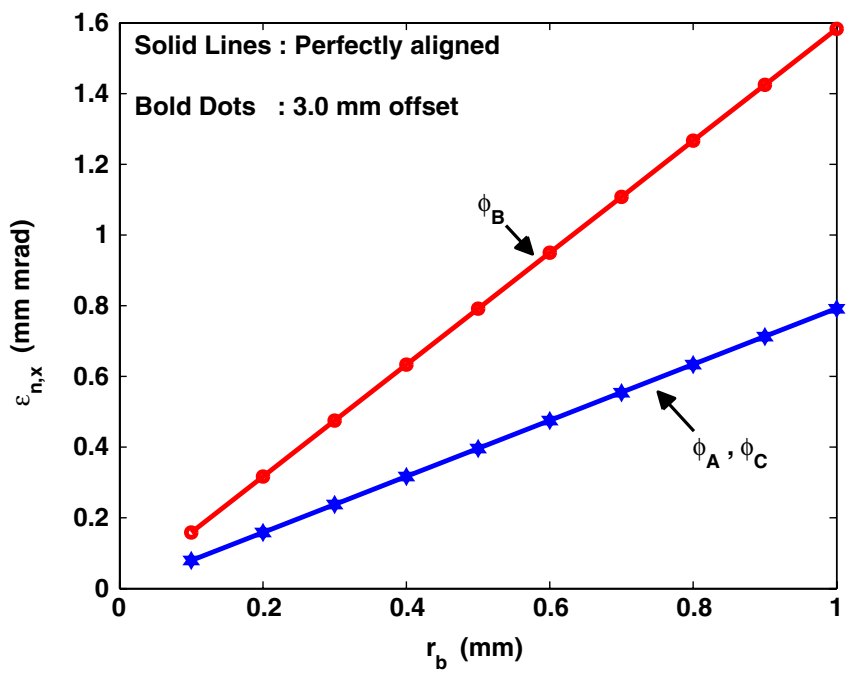

FIG. 19. Vertical projected emittance of beam for different bunch radius. Solid lines correspond to perfect alignment and the bold dots are the representative of " $3 \mathrm{~mm}$ " misalignment.

of horizontal misalignment for the given engineering requirement.

\section{B. Beam projected emittance for multiple cavities}

In this section we compare the effect of bunch radius on beam projected emittance between aligned and misaligned cavities. Figure 19 shows the evolution of the transverse projected emittance for different bunch radius and its comparison between aligned and misaligned arrangements. For the sake of completeness, the comparison is made for the undeflected and the deflected beams. As expected, we observe the linear behavior and the graphs 
involving aligned and misaligned cavities are superimposed. This study confirms the reliability of normal conducting cavity arrangement against the misalignment much beyond the specified tolerance level. Moreover, the projected normalized rms emittance dilution is $0.158 \mathrm{~mm}$ mrad to that of $0.15 \mathrm{~mm} \mathrm{mrad}$ of superconducting structurealmost the same. In brief, we can conclude that both the normal and superconducting structures are equally good in EM properties and beam dynamics.

\section{CONCLUSION}

In this paper, we report the beam dynamics studies for TEM-type rf separators in order to separate a $1497 \mathrm{MHz}$ $11 \mathrm{GeV}$ beam into three $499 \mathrm{MHz}$ variable intensity beams for the purpose of delivering to three experimental stations simultaneously. Three-dimensional tracking studies of novel superconducting and an array of existing CEBAF normal conducting rf separators have been performed. We have examined the performance of these structures (NC and SC) in order to verify the specific requirements confirming their suitability. The nonlinear effect of EM fields inside the cavities affect the dynamics of the particle's trajectory only within the cavity and their immediate vicinities, however, the effect becomes unimportant in the region of our interest. Moreover, the finite thickness of these cavities results in the finite transit time, which in turn causes nonzero residual orbit offset to the reference beam directed to hall $\mathrm{B}$. This effect is shown to be unimportant for the application of our interest. Furthermore, the horizontal and vertical misalignments in the series arrangement of normal conducting structures are very well tolerable against mechanical vibrations due to strong concentration of EM fields between the rods. The beam clear condition is very well satisfied by the superconducting structure due to the large size of beam pipe aperture, which in the case of $\mathrm{NC}$ cavity is fulfilled by a series combinations of six 2-cell structures. Nevertheless, the emittance dilution as a result of beam deflection justifies the specification. In brief, we conclude that the overall performance of both the $\mathrm{NC}$ and the SC cavities are satisfactory.

\section{ACKNOWLEDGMENTS}

This manuscript has been authored by Jefferson Science Associates, L. L.C. under U.S. DOE Contract No. DEAC05-06OR23177. The authors would like to thank CST support team for their valuable help in CST simulations.

[1] S. U. De Silva and J. R. Delayen, in Proceedings of the 2010 Linear Accelerator Conference LINAC2010, Tsukuba, Japan, 2010, p. 812.

[2] J. R. Delayen and H. Wang, Phys. Rev. ST Accel. Beams 12, 062002 (2009).

[3] C. Leemann and C.G. Yao, JLAB Technical Report No. CEBAF TN-90-217, 1990.

[4] L. T. C. Hovater, G. Arnold, J. Fugitt, L. Harwood, R. Kazimi, G. Lathi, J. Mammosser, R. Nelson, and C. Piller, JLAB Report No. JLAB-ACC-96-18, 1996.

[5] S. Ahmed, G. A. Krafft, K. Deitrick, S. U. De Silva, J.R. Delayen, M. Spata, M. Tiefenback, A. Hofler, and K. Beard, in Proceedings of the 2011 Particle Accelerator Conference, NY, USA (IEEE, New York, 2011), pp. 790-792.

[6] R. Kazimi, J. Fugitt, A. Krycuk, C. Sinclair, and L. Turlington, in Proceedings of the Particle Accelerator Conference, Washington, DC, 1993 (IEEE, New York, 1993), p. 1109.

[7] General particle tracer (GPT), Release 2.82 ed. Pulsar Physics, Soest, The Netherlands [http://www.pulsar.nl/ gpt].

[8] T. J. Roberts and D. M. Kaplan, in Proceedings of the 2007 Particle Accelerator Conference, Albuquerque, New Mexico (IEEE, New York, 2007) [http://g4beamline .muonsinc.com].

[9] CST Particle Studio (CST PS), Release 2010 ed. CST GmbH, 64289 Darmstadt, Germany [http://www.cst.com].

[10] S. Agostinelli et al., Nucl. Instrum. Methods Phys. Res., Sect. A 506, 250 (2003) [http://geant4.cern.ch].

[11] J. Shi, H. Chen, C. Tang, S. Zheng, W. Huang, J. Power, C. Jing, K. Kim, W. Gai, and D. Li, Nucl. Instrum. Methods Phys. Res., Sect. A 598, 388 (2009).

[12] G. A. Krafft, in JLab Technical Note No. CEBAF-TN-92008, 1992. 